\title{
Planar and Quasi Planar Simultaneous Geometric Embedding ${ }^{\star}$
}

\author{
Emilio Di Giacomo ${ }^{1}$, Walter Didimo ${ }^{1}$, Giuseppe Liotta ${ }^{1}$, \\ Henk Meijer ${ }^{2}$, and Stephen Wismath ${ }^{3}$ \\ 1 Dipartimento di Ingegneria, Università degli Studi di Perugia, Italy \\ \{emilio.digiacomo, walter.didimo, giuseppe.liotta\}@unipg.it \\ ${ }^{2}$ University College Roosevelt, The Netherlands \\ h.meijer@ucr.nl \\ 3 Department of Mathematics and Computer Science, University of Lethbridge, Canada \\ wismath@uleth.ca
}

\begin{abstract}
A simultaneous geometric embedding ( $S G E)$ of two planar graphs $G_{1}$ and $G_{2}$ with the same vertex set is a pair of straight-line planar drawings $\Gamma_{1}$ of $G_{1}$ and $\Gamma_{2}$ of $G_{2}$ such that each vertex is drawn at the same point in $\Gamma_{1}$ and $\Gamma_{2}$. Many papers have been devoted to the study of which pairs of graphs admit a SGE, and both positive and negative results have been proved. We extend the study of SGE, by introducing and characterizing a new class of planar graphs that makes it possible to immediately extend several positive results that rely on the property of strictly monotone paths. Moreover, we introduce a relaxation of the SGE setting where $\Gamma_{1}$ and $\Gamma_{2}$ are required to be quasi planar (i.e., they can have crossings provided that there are no three mutually crossing edges). This relaxation allows for the simultaneous embedding of pairs of planar graphs that are not simultaneously embeddable in the classical SGE setting and opens up to several new interesting research questions.
\end{abstract}

\section{Introduction}

The simultaneous embedding ( $S E$ ) problem is one of the most studied in Graph Drawing since the publication of the first seminal results on the subject by Braß et al. [56]. Given two planar graphs with the same vertex set $G_{1}=\left(V, E_{1}\right)$ and $G_{2}=\left(V, E_{2}\right)$, the SE problem asks whether a planar drawing $\Gamma_{1}$ of $G_{1}$ and a planar drawing $\Gamma_{2}$ of $G_{2}$ exist such that each vertex of $V$ is drawn at the same point in $\Gamma_{1}$ and $\Gamma_{2}$. If so, pair $\left\langle\Gamma_{1}, \Gamma_{2}\right\rangle$ is called a simultaneous embedding $(S E)$ of $\left\langle G_{1}, G_{2}\right\rangle$. Several variants and generalizations of the SE problem (e.g., extensions to $k>2$ graphs) have also been studied. A comprehensive survey on SE can be found in [4].

In this paper we concentrate on the most desirable, but also the most restrictive, setting of the SE problem, namely the simultaneous geometric embedding ( $S G E$ ) setting, where drawings $\Gamma_{1}$ and $\Gamma_{2}$ are required to have straight-line edges. A paper by Angelini et al. [3] establishes that there exist a tree of depth four and a path that do not admit a

\footnotetext{
* Research supported in part by the MIUR project AMANDA "Algorithmics for MAssive and Networked DAta", prot. 2012C4E3KT_001. 
SGE. On the positive side, a path can always be simultaneously embedded with a tree of depth at most two [3] and with other kinds of trees such as caterpillars (i.e., trees that become paths after the removal of the degree-one vertices) or stars (trees with at most one vertex of degree greater than one) and their extensions (see e.g., [6]). Other positive results involve simple types of cyclic graphs (see, e.g., [67]).

Most of the positive results about SGE rely on reducing one of the two graphs $G_{1}$ or $G_{2}$ to a path that is realized in a strictly monotone fashion. The fundamental property of a strictly monotone drawing of a path, say in the $y$-direction, is that it is planar independently of the $x$-coordinates given to the vertices. This property makes it possible to arbitrarily assign the $x$-coordinates of the other graph when looking for a SGE. Motivated by this observation, Fowler and Kobourov characterized the class of graphs that can be simultaneously embedded with any given path drawn in a strictly monotone way [11]; they call these graphs the Unlabeled Level Planar (ULP) graphs. A characterization for ULP trees was previously given in [9].

In this paper we extend the study of SGE in two different directions:

(i) We characterize the class of graphs that have the same property as strictly monotone paths; namely, the planar graphs such that there exists a suitable $y$-leveling of the vertices for which a planar drawing exists for any $x$-leveling of the vertices. We prove that this class, which we term EAP 1 , is a proper sub-class of the ULP; every graph that can be simultaneously embedded with a strictly monotone path is also simultaneously embeddable with an EAP graph, hence our finding immediately enlarges the set of positive results on SGE. We also extend the result in [3], proving that EAP graphs can always be simultaneously embedded with a tree of depth at most two. We remark that the SGE technique in [3] does not rely on a strictly monotone drawing of the path.

(ii) Since SGE is a rather restricting setting, we study simultaneous geometric embeddings where both $\Gamma_{1}$ and $\Gamma_{2}$ are required to be "nearly planar" in some sense. Namely, we require that each of the two drawings is quasi planar, i.e., it does not contain three mutually crossing edges. We remark that quasi planar drawings have been widely studied in the literature (see, e.g., [12|12 13]); they fall into a line of research called "beyond planarity", which is receiving lots of interest in the graph drawing community. For simultaneous geometric quasi planar embedding (SGQPE) setting we generalize the ULP and the EAP graph classes, thus obtaining several positive results; for example, we prove that a tree and a path always admit a SGQPE in contrast to the negative result in [3] for the SGE setting. More in general we prove that every tree has a SGQPE with a meaningful subfamily of the outerplanar graphs.

The results of point $(i)$ are presented in Sec. 3 and those of point $(i i)$ are in Sec. 4. Preliminaries are in Sec. 2. Conclusions and open problems are in Sec.5.

\section{Preliminaries}

Let $\left\langle G_{1}=\left(V, E_{1}\right), G_{2}=\left(V, E_{2}\right)\right\rangle$ be a pair of planar graphs with the same vertex set. A simultaneous geometric embedding $(S G E)$ of $\left\langle G_{1}, G_{2}\right\rangle$ is a pair of drawings $\left\langle\Gamma_{1}, \Gamma_{2}\right\rangle$

\footnotetext{
${ }^{1}$ The explanation of this acronym is clarified in the paper. The same class of graphs is defined with the name of column planar graphs in [10].
} 
such that: $(i) \Gamma_{i}$ is a planar straight-line drawing of $G_{i}$ for $i=1,2 ;(i i)$ each vertex $v \in V$ is represented by the same point in $\Gamma_{1}$ and $\Gamma_{2}$.

Let $G$ be a planar graph. An $x$-leveling of $G$ is a mapping $\mathcal{X}: V \rightarrow \mathbb{R}$ that assigns to each vertex of $G$ a distinct real $x$-coordinate. A $y$-leveling of $G$ is a mapping $\mathcal{Y}: V \rightarrow \mathbb{R}$ that assigns to each vertex of $G$ a distinct real $y$-coordinate. Let $G$ be a planar graph with a given $x$-leveling $\mathcal{X}$ and a given $y$-leveling $\mathcal{Y}$. We denote by $\Gamma(\mathcal{X}, \mathcal{Y})$ a straightline drawing of $G$ obtained by drawing each vertex $v$ at point $(\mathcal{X}(v), \mathcal{Y}(v))$ and each edge as a straight-line segment between its endpoints. If $\Gamma(\mathcal{X}, \mathcal{Y})$ has no three collinear vertices we say that $\mathcal{X}$ is general with respect to $\mathcal{Y}$, and $\mathcal{Y}$ is general with respect to $\mathcal{X}$.

Let $G$ be a graph and let $\Gamma$ be a straight-line drawing of $G$. Two edges of $G$ are independent if they do not have an endvertex in common. The independent horizontal stabbing number of $\Gamma$, denoted by ihs $(\Gamma)$, is the maximum number of independent edges of $\Gamma$ intersected by a horizontal line. The independent horizontal stabbing number of $G$, denoted by ihs $(G)$, is the minimum independent horizontal stabbing number over all straight-line drawings of $G$. A horizontal line is called a stabber; the stabber of equation $y=l$ is the stabber at $l$. Two drawings with the same top-to-bottom order of the vertices have the same independent horizontal stabbing number. The next lemmas are used to remove collinear points in a drawing.

Lemma 1. Let $G$ be a graph. Let $\mathcal{Y}$ be a $y$-leveling of $G$ and let $\mathcal{X}$ be an $x$-leveling of $G$ that is not general with respect to $\mathcal{Y}$. There exists an $x$-leveling $\mathcal{X}^{\prime}$ that is general with respect to $\mathcal{Y}$ and such that if two edges cross in $\Gamma\left(\mathcal{X}^{\prime}, \mathcal{Y}\right)$ then they cross in $\Gamma(\mathcal{X}, \mathcal{Y})$.

Lemma 2. Let $G$ be a graph. Let $\mathcal{Y}$ be a y-leveling of $G$ and let $\mathcal{X}$ be an $x$-leveling of $G$ that is not general with respect to $\mathcal{Y}$. There exists a $y$-leveling $\mathcal{Y}^{\prime}$ that is general with respect to $\mathcal{X}$ and such that ihs $\left(\Gamma\left(\mathcal{X}, \mathcal{Y}^{\prime}\right)\right)=\operatorname{ihs}(\Gamma(\mathcal{X}, \mathcal{Y}))$.

\section{EAP Graphs and Simultaneous Geometric Embedding}

Before defining EAP graphs, we recall the definition of ULP graphs (introduced in [11]), which we rename as $A E P$ graphs. Let $G$ be a planar graph. $G$ is an $A E P$ graph if for any $y$-leveling $\mathcal{Y}$ of $G$, there exists an $x$-leveling $\mathcal{X}$ such that $\Gamma(\mathcal{X}, \mathcal{Y})$ is planar. $G$ is an $E A P$ graph if there exists a $y$-leveling $\mathcal{Y}$ of $G$, called universal $y$-leveling, such that for any $x$-leveling $\mathcal{X}$ that is general with respect to $\mathcal{Y}, \Gamma(\mathcal{X}, \mathcal{Y})$ is planat 2 . We denote by $A E P$ the set of AEP graphs and by EAP the set of EAP graphs. Note that, in the definition of EAP graphs we consider only $x$-levelings that are general with respect to the $y$-leveling $\mathcal{Y}$. This restriction is necessary since otherwise no graphs other than paths could be EAP. Namely, if three collinear points are allowed, then for any given $y$-leveling there would exist an $x$-leveling such that the resulting drawing has edges that overlap each other. These trivial counterexamples are avoided by restricting the definition to those $x$-levelings that do not cause three collinear points. The interplay between AEP and

\footnotetext{
${ }^{2}$ The names AEP and EAP are acronyms coming from the definitions of the AEP and EAP graphs, respectively. Namely, a graph is an AEP graph if "for Any $y$-leveling, there Exists an $x$-leveling such that the resulting drawing is Planar", while a graph is EAP if "there Exists a $y$-leveling such that for Any $x$-leveling the resulting drawing is Planar".
} 
EAP graphs makes it possible to extend several results about SGE previously described in the literature, thanks to the following result (see also Theorem 2 and Corollary 2).

Theorem 1. Let $\left\langle G_{1}, G_{2}\right\rangle$ be a pair of graphs such that $G_{1} \in A E P$ and $G_{2} \in E A P$. Then $\left\langle G_{1}, G_{2}\right\rangle$ admits a $S G E$.

Proof. Since $G_{2}$ is an EAP graph, there exists a universal $y$-leveling $\mathcal{Y}$ of $G_{2}$. Consider $\mathcal{Y}$ as a $y$-leveling of $G_{1}$; we aim at finding an $x$-leveling $\mathcal{X}$ of $G_{1}$ that is general with respect to $\mathcal{Y}$ and such that $\Gamma(\mathcal{X}, \mathcal{Y})$ is planar. Since $G_{1} \in A E P$, then there exists an $x$-leveling $\mathcal{X}^{\prime}$ such that $\Gamma_{1}\left(\mathcal{X}^{\prime}, \mathcal{Y}\right)$ is a planar drawing of $G_{1}$. If $\mathcal{X}^{\prime}$ is general with respect to $\mathcal{Y}$ we set $\mathcal{X}=\mathcal{X}^{\prime}$; if $\mathcal{X}^{\prime}$ is not general with respect to $\mathcal{Y}$, then, by Lemma 1 , there exists an $x$-leveling $\mathcal{X}^{\prime \prime}$ that is general with respect to $\mathcal{Y}$ and such that $\Gamma\left(\mathcal{X}^{\prime \prime}, \mathcal{Y}\right)$ is planar; in this case we set $\mathcal{X}=\mathcal{X}^{\prime \prime}$. In either case we have the desired $x$-leveling $\mathcal{X}$. Consider $\mathcal{X}$ as an $x$-leveling for $G_{2}$. Then $\Gamma_{2}(\mathcal{X}, \mathcal{Y})$ is a planar drawing of $G_{2}$ because $G_{2}$ is an EAP graph, $\mathcal{Y}$ is a universal $y$-leveling of $G_{2}$ and $\mathcal{X}$ is general with respect to $\mathcal{Y}$. Thus, $\left\langle\Gamma_{1}(\mathcal{X}, \mathcal{Y}), \Gamma_{2}(\mathcal{X}, \mathcal{Y})\right\rangle$ is a SGE of $\left\langle G_{1}, G_{2}\right\rangle$.

Characterization of EAP graphs. AEP graphs have been characterized by Fowler and Kobourov [11]. We first show that EAP graphs constitute a subfamily of AEP graphs with stronger properties (Lemma 3) and then we characterize EAP graphs (Theorem 2).

Lemma 3. Let $G \in E A P$. Then $G \in A E P$.

Proof. Let $\mathcal{Y}^{\prime}$ be an arbitrary given $y$-leveling of $G$. In order to prove that $G \in A E P$ we must show that there exists an $x$-leveling $\mathcal{X}^{\prime}$ such that $\Gamma\left(\mathcal{X}^{\prime}, \mathcal{Y}^{\prime}\right)$ is a planar drawing. Since $G$ is an EAP graph, there exists a universal $y$-leveling $\mathcal{Y}$ of $G$. We define an $x$ leveling $\mathcal{X}$ for $G$ as $\mathcal{X}=\mathcal{Y}^{\prime}$. In other words we assign to each vertex $v$ an $x$-coordinate $\mathcal{X}(v)$ that is equal to the $y$-coordinate $\mathcal{Y}^{\prime}(v)$ assigned to $v$ by $\mathcal{Y}^{\prime}$. We have two cases:

Case 1: $\mathcal{X}$ is general with respect to $\mathcal{Y}$. In this case, since $G \in E A P, \Gamma(\mathcal{X}, \mathcal{Y})$ is a planar drawing. If we set $\mathcal{X}^{\prime}=\mathcal{Y}$, we have that $\Gamma\left(\mathcal{X}^{\prime}, \mathcal{Y}^{\prime}\right)$ is the drawing $\Gamma(\mathcal{X}, \mathcal{Y})$ rotated by $90^{\circ}$ and hence it is planar.

Case 2: $\mathcal{X}$ is not general with respect to $\mathcal{Y}$. By Lemma 2 there exists a $y$-leveling $\mathcal{Y}^{\prime \prime}$ that is general with respect to $\mathcal{X}$ such that ihs $\left(\Gamma\left(\mathcal{X}, \mathcal{Y}^{\prime \prime}\right)\right)=\operatorname{ins}(\Gamma(\mathcal{X}, \mathcal{Y}))$. Let $\mathcal{X}^{\prime \prime}$ be an $x$-leveling that is general with respect to $\mathcal{Y}$; we have ihs $(\Gamma(\mathcal{X}, \mathcal{Y}))=\operatorname{ins}\left(\Gamma\left(\mathcal{X}^{\prime \prime}, \mathcal{Y}\right)\right)$ because $\Gamma(\mathcal{X}, \mathcal{Y})$ and $\Gamma\left(\mathcal{X}^{\prime \prime}, \mathcal{Y}\right)$ have the same $y$-leveling. Since $G$ is an EAP graph, $\operatorname{ins}\left(\Gamma\left(\mathcal{X}^{\prime \prime}, \mathcal{Y}\right)\right)=1$ and hence ihs $\left(\Gamma\left(\mathcal{X}, \mathcal{Y}^{\prime \prime}\right)\right)=1$, which implies that $\Gamma\left(\mathcal{X}, \mathcal{Y}^{\prime \prime}\right)$ is planar (otherwise its independent horizontal stabbing number would be at least two). If we set $\mathcal{X}^{\prime}=\mathcal{Y}^{\prime \prime}$ then $\Gamma\left(\mathcal{X}^{\prime}, \mathcal{Y}^{\prime}\right)$ is $\Gamma\left(\mathcal{X}, \mathcal{Y}^{\prime \prime}\right)$ rotated by $90^{\circ}$, and hence is planar.

In both cases we have found an $x$-leveling $\mathcal{X}^{\prime}$ such that $\Gamma\left(\mathcal{X}^{\prime}, \mathcal{Y}^{\prime}\right)$ is a planar drawing. Since $\mathcal{Y}^{\prime}$ is arbitrary, we have that $G \in A E P$.

By Lemma 3 we have that $E A P \subseteq A E P$. By the characterization of Fowler and Kobourov [11] we know precisely the graphs in $A E P$; therefore in order to characterize the class $E A P$ we can establish which graphs of the set $A E P$ are EAP graphs. The family of AEP graphs is the union of the following three families: radius-2 stars, extended degree-3 spiders, and generalized caterpillars. In order to recall the definition of these families we start by defining four gadgets each having two vertices called poles. 


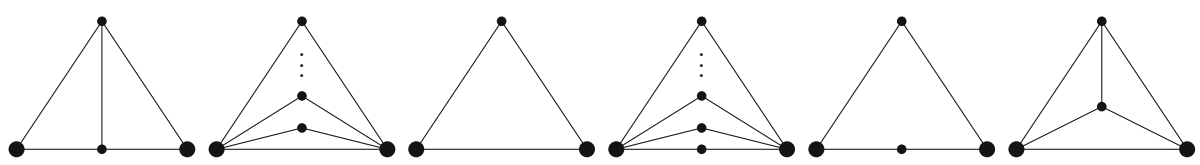

(a) Kite gadget (b) $K_{3}^{*}$ gadget (c) $K_{3}$ gadget (d) $C_{4}^{+}$gadget (e) $C_{4}$ gadget (f) $K_{4}$ gadget

Fig. 1. Illustration of the different gadgets. Bigger vertices are the poles of the gadget

Given an edge $e$ of a graph, replacing $e$ with one of the gadgets means to replace $e$ with the gadget so that the end-vertices of $e$ coincide with the two poles of the gadget. The kite gadget is a 4-cycle plus an edge connecting the two vertices that are not poles (Fig. 1(a)). A $K_{3}^{*}$ gadget is a set of $k \geq 13$-cycles sharing the two poles and the edge connecting them (Fig. 1(b)]. A $K_{3}^{*}$ gadget consisting of a single cycle is called a $K_{3}$ gadget (Fig. 1(c)]. A $C_{4}^{+}$gadget is a set of $k \geq 2$ paths with two edges, connecting the two poles (Fig.[1(d)]. A $C_{4}^{+}$gadget consisting of exactly two paths is called a $C_{4}$ gadget (Fig. 1(e). A $K_{4}$ gadget is the complete graph on four vertices (Fig. 1(f)]. The poles of a $K_{4}$ gadget are any two of its vertices. A star is a graph $K_{1, k}$ for some $k \geq 3$. A radius-2 star is a star in which at least one edge has been subdivided once (see Fig. 2(a). A degree-3 spider is an arbitrary subdivision of $K_{1,3}$. A 1-connected extended degree-3 spider is a degree- 3 spider with two optional additional edges: an edge connecting two vertices adjacent to the unique degree- 3 vertex and an edge connecting two leaves (see Fig. 2(b). A 2-connected extended degree-3 spider is either a cycle or a cycle where an edge is replaced by a $K_{3}$ gadget, a $C_{4}$ gadget, or a kite gadget (see Fig. 2(c)). An extended degree-3 spider is either a 1-connected extended degree-3 spider or a 2-connected extended degree-3 spider. A caterpillar is a tree such that removing all leaves we get a path, called the spine of the caterpillar. A generalized caterpillar is a caterpillar in which each edge of the spine can be replaced by a $K_{3}^{*}$ gadget, or a $C_{4}^{+}$gadget, or a kite gadget, and for each endvertex $u$ of the spine, one edge connecting $u$ to a leaf can be replaced by a $K_{3}^{*}$ gadget, or a $C_{4}^{+}$gadget, or a kite gadget, or a $K_{4}$ gadget (see Fig. 2(d)).

We define a new family of graphs that we call fat caterpillars and that is a subfamily of generalized caterpillars. A fat caterpillar is a graph obtained from a caterpillar by replacing some of the edges of the spine with a $K_{3}$ gadget (see Fig. 2(e). Notice that fat caterpillars are exactly the generalized caterpillars with no cycle of length larger than three. Let $G^{\prime}$ be the subgraph of $G$ obtained by removing all degree-one vertices; $G^{\prime}$ consists of a path $\Pi=\left(v_{1}, v_{2}, \ldots, v_{k}\right)$ plus a set of vertices each adjacent to two consecutive vertices of $\Pi$. The vertices of $\Pi$ are called the path vertices of $G$; in particular $v_{1}$ and $v_{k}$ are called extreme path vertices. The remaining vertices of $G^{\prime}$ are called the tip vertices of $G$; a tip vertex that is adjacent to $v_{i}$ and $v_{i+1}$ will be denoted by $u_{i}$. Each vertex of $G$ of degree one is adjacent to a path vertex. The degree-one vertices adjacent to $v_{i}$ will be denoted by $w_{i, j}$, with $j=1,2, \ldots, h_{i}$, where $h_{i} \geq 0$. We start with a technical lemma that will be used to characterize EAP graphs.

Lemma 4. A graph $G$ is an EAP graph if and only if ihs $(G)=1$.

The next lemma can be proved by showing that, in each case, ihs $(G)>1$, which, by Lemma 4, implies that $G \notin E A P$. 


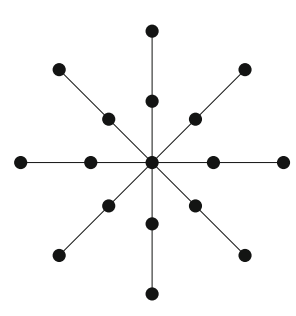

(a)

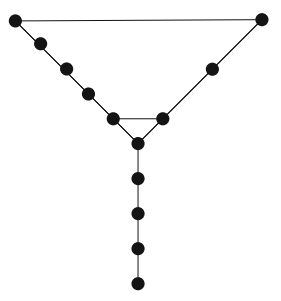

(b)

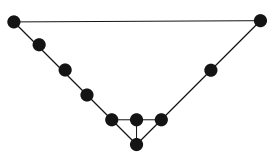

(c)

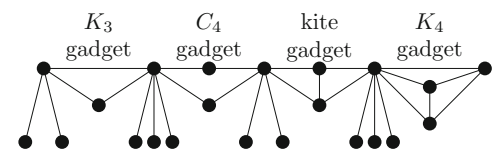

(d)

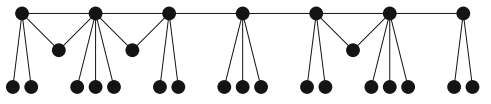

(e)

Fig. 2. (a) A radius-2 star. (b) A 1-connected extended degree-3 spider. (c) A 2-connected extended degree-3 spider (containing a kite gadget). (d) A generalized caterpillar. (e) A fat caterpillar.

Lemma 5. Let $G$ be a graph such that either: $(i) G$ is a radius-2 star that is not a generalized caterpillar; or (ii) G contains a cycle of length at least 4; or (iii) $G$ is an extended degree-3 spider that is not a generalized caterpillar. Then $G \notin E A P$.

Theorem 2. A planar graph $G$ is an EAP graph if and only if it is a fat caterpillar.

Proof. "Only if part". If $G$ is an EAP graph, then by Lemma 3 it is also AEP and therefore it is either a radius-2 star, or an extended degree-3 spider, or a generalized caterpillar. By Lemma 5 must be a generalized caterpillar and cannot contain a cycle of length four. Hence it must be a fat caterpillar.

"If part". Let $G$ be a fat caterpillar. We prove that ihs $(G)=1$. We define a $y$-leveling $\mathcal{Y}$ of $G$ as follows. For each path vertex $v_{i}(1 \leq i \leq k)$ of $G$ we set $\mathcal{Y}\left(v_{i}\right)=2 i$. For each tip vertex $u_{i}(1 \leq i \leq k)$ of $G$ we set $\mathcal{Y}\left(u_{i}\right)=\mathcal{Y}\left(v_{i}\right)+1$. For each degree-one vertex $w_{i, j}$ we set $\mathcal{Y}\left(w_{i, j}\right)=\mathcal{Y}\left(v_{i}\right)+\frac{j}{h_{i}+1}\left(j=1,2, \ldots, h_{i}\right)$. Observe that the vertices of $G$ have been assigned a different value and therefore $\mathcal{Y}$ is a valid $y$-leveling. Consider now an arbitrary $x$-leveling $\mathcal{X}$. We show that $\operatorname{ihs}(\Gamma(\mathcal{X}, \mathcal{Y}))=1$, which implies that ihs $(G)=1$. Consider a stabber $\ell$ at $l, l \in \mathbb{R}$. If $l<2$ or $l>2 k$, then $\ell$ does not intersect any edge of $\Gamma(\mathcal{X}, \mathcal{Y})$. If $l=2 i$ for $1 \leq i \leq k$, then $\ell$ passes through vertex $v_{i}$ and it intersects only the edges incident to $v_{i}$, which are not independent. If $l=2 i+1$ for $1 \leq i \leq k-1$, then $\ell$ either intersects only the edge $\left(v_{i}, v_{i+1}\right)$ (if the tip vertex $u_{i}$ does not exist), or it intersects the three edges $\left(v_{i}, v_{i+1}\right),\left(v_{i}, u_{i}\right)$, and $\left(u_{i}, v_{i+1}\right)$ (if the tip vertex $u_{i}$ exists) no two of which are independent. If $2 i<\ell<2 i+1$ for $1 \leq i \leq k-1$, then $\ell$ intersects the edge $\left(v_{i}, v_{i+1}\right)$, possibly the edge $\left(v_{i}, u_{i}\right)$, and possibly some of the edges $\left(v_{i}, w_{i, j}\right)\left(j=1,2, \ldots, h_{i}\right)$. No two of these edges are independent. Finally, if $2 i+1<l<2 i+2$ for $1 \leq i \leq k-1$, then $\ell$ intersects the edge $\left(v_{i}, v_{i+1}\right)$ and possibly the edge $\left(u_{i}, v_{i+1}\right)$, which are not independent. Thus, no stabber intersects two independent edges in $\Gamma(\mathcal{X}, \mathcal{Y})$ and therefore ihs $(\Gamma(\mathcal{X}, \mathcal{Y}))=1$. It follows that ihs $(G)=1$ and, by Lemma4, $G \in E A P$. 


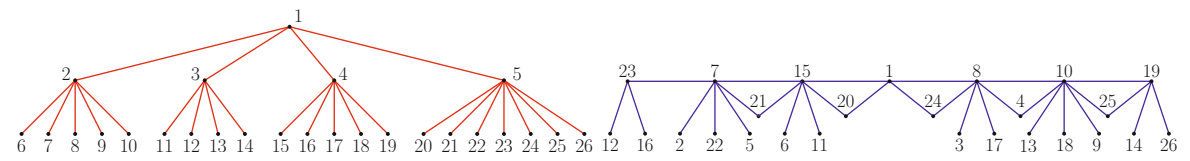

(a)

(b)

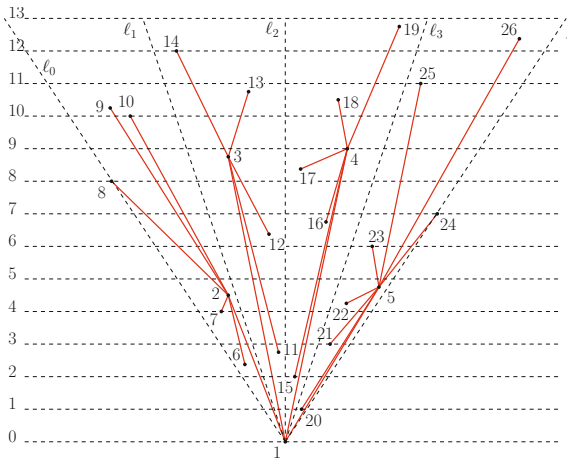

(c)

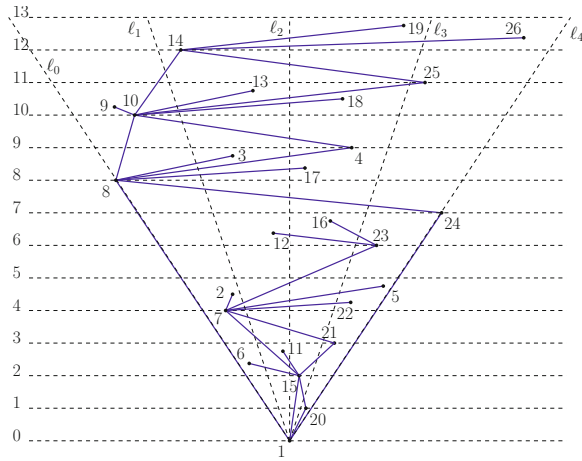

(d)

Fig. 3. (a)-(b) A pair of graphs $\left\langle G_{1}, G_{2}\right\rangle$; $G_{1}$ is a tree of depth two while $G_{2}$ is a fat caterpillar. $G_{1}$ and $G_{2}$ have the same vertex set; each vertex has the same label in (a) and (b). (c)-(d) A simultaneous embedding of $\left\langle G_{1}, G_{2}\right\rangle$.

An immediate consequence of Lemma 3 and Theorem 2 is that $E A P$ is properly contained in AEP (Corollary[1). Theorems 1 and 2 imply Corollary 2.

Corollary 1. $E A P \subset A E P$.

Corollary 2. Let $\left\langle G_{1}, G_{2}\right\rangle$ be a pair of graphs such that $G_{1}$ is either a radius-2 star, an extended degree-3 spider or a generalized caterpillar, and $G_{2}$ is a fat caterpillar. Then $\left\langle G_{1}, G_{2}\right\rangle$ admits a $S G E$.

The characterization of Theorem 2 implies that all planar graphs that are known to be simultaneously embeddable with a strictly monotone path [4] are in fact simultaneously embeddable with a fat caterpillar (i.e., EAP graphs). Moreover, EAP graphs open up two further research directions. A first research direction is to extend to fat caterpillars other existing SGE results that involve paths that are not realized in a strictly monotone fashion. A second research direction is to extend the result of Theorem 1 to simultaneous drawings that are not planar but where some crossing configurations are forbidden. Regarding the first research direction, we prove below that fat caterpillars can be used to generalize a result by Angelini et al. [3] in which it is proved that a path and a tree of depth two always admit a SGE. As for "nearly planar" simultaneous geometric embeddings, we devote Sec. 4 to quasi planar drawings.

Theorem 3. Let $\left\langle G_{1}, G_{2}\right\rangle$ be a pair of graphs such that $G_{1}$ is a tree of depth 2 and $G_{2}$ is a fat caterpillar. Then $\left\langle G_{1}, G_{2}\right\rangle$ admits a $S G E$.

Sketch of Proof: The technique is inspired by the one described in [3]. Since $G_{1}$ is a tree of depth 2 , removing the root of $G_{1}$ we obtain a set of stars $S_{1}, S_{2}, \ldots, S_{h}$. Let 
$\ell_{0}, \ell_{1}, \ldots, \ell_{h}$ be a set of half-lines each having initial point $(0,0)$ and all extending in the upper half-plane, ordered as they are encountered rotating clockwise starting at the negative $x$-axis. Let $C_{i}$ be the cone delimited by $\ell_{i-1}$ and $\ell_{i}$. In order to obtain a planar drawing of a star it is sufficient to map its vertices to a set of points in general position, i.e., a set of points such that there are no three collinear points. So in order to draw $G_{1}$ we can map the vertices of each $S_{i}(i=1,2, \ldots, h)$ to points in general position of a different cone $C_{j}$ and the root of $G_{1}$ to point $(0,0)$ shared by all regions. In this way each star $S_{i}$ is drawn planarly inside a different $C_{j}$ (thus different stars do not cross each other) and the root vertex can be connected without crossings to its adjacent vertex of each star $S_{i}$ (see Fig. 3(c) ). It can be shown that $G_{2}$ admits a drawing such that the vertex corresponding to the root of $G_{1}$ is mapped to $(0,0)$ and the points corresponding to each star $S_{i}$ are mapped to a different cone $C_{j}$ (see Fig. 3(d).

\section{Simultaneous Geometric Quasi Planar Embeddings}

We introduce and study geometric simultaneous embeddings where each of the two drawings are not required to be planar but only quasi planar. We implicitly assume that all drawings are straight-lines. Let $G$ be a graph and let $\Gamma$ be a drawing of $G$. $\Gamma$ is quasi planar if there are no three mutually crossing edges. A graph is quasi planar if it admits a quasi planar drawing. Let $\left\langle G_{1}=\left(V, E_{1}\right), G_{2}=\left(V, E_{2}\right)\right\rangle$ be a pair of quasi planar graphs with the same vertex set. A simultaneous geometric quasi planar embedding (SGQPE) of $\left\langle G_{1}, G_{2}\right\rangle$ is a pair of drawings $\left\langle\Gamma_{1}, \Gamma_{2}\right\rangle$ such that: $(i) \Gamma_{i}$ is a quasi planar straight-line drawing of $G_{i}$ for $i=1,2$; $(i i)$ each vertex $v \in V$ is represented by the same point in $\Gamma_{1}$ and $\Gamma_{2}$. We extend the definition of AEP and EAP graphs to the quasi planar case as follows. A quasi planar graph $G$ is an $A E Q P$ graph if for any $y$-leveling $\mathcal{Y}$ of $G$, there exists an $x$-leveling $\mathcal{X}$ such that $\Gamma(\mathcal{X}, \mathcal{Y})$ is quasi planar. $G$ is an $E A Q P$ graph if there exists a $y$-leveling $\mathcal{Y}$ of $G$, called a universal quasi planar leveling, such that for any $x$-leveling $\mathcal{X}$ that is general with respect to $\mathcal{Y}, \Gamma(\mathcal{X}, \mathcal{Y})$ is quasi planar. Denote by $A E Q P$ and $E A Q P$ the set of AEQP and EAQP graphs, respectively. The next result generalizes Theorem 1

Theorem 4. Let $\left\langle G_{1}, G_{2}\right\rangle$ be a pair of graphs such that $G_{1} \in A E Q P$ and $G_{2} \in$ $E A Q P$. Then $\left\langle G_{1}, G_{2}\right\rangle$ admits a SGQPE.

Motivated by Theorem 4 we study the interplay between AEQP and EAQP graphs and also their relationships with AEP and EAP graphs, which are summarized by Fig. 4 and Theorem 6 We first show that any AEP graph is an EAQP graph (Lemma 7). The next technical lemma generalizes Lemma 4 (the proof is similar).

Lemma 6. A graph $G$ is an EAQP graph if and only if ihs $(G) \leq 2$.

Lemma 7. $A E P \subset E A Q P$.

Sketch of Proof: Let $G$ be an $A E P$ graph; it is either a radius-2 star, or a generalized degree-3 spider, or a generalized caterpillar. For each type of graph we describe a $y$ leveling that is universal quasi planar. The proof of correctness is omitted. 


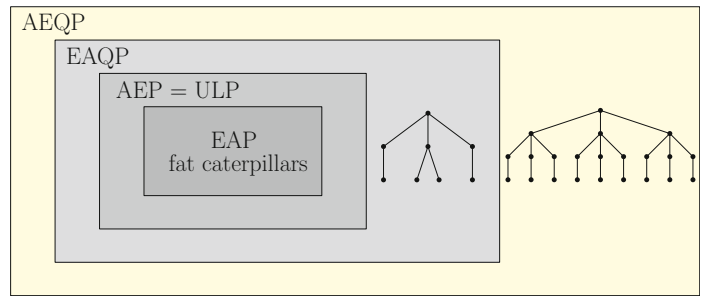

Fig. 4. Proper inclusions among the different classes of graphs studied in this paper

Radius-2 Star. (see Fig. 5(a) . Let $G$ be a radius-2 star and let $v$ be the unique vertex of $G$ with degree larger than two. Denote by $u_{1}, u_{2}, \ldots, u_{k}$ the neighbors of $v$ and denote by $w_{i}$ the neighbor of $u_{i}$ different from $v$ (if it exists). We define a $y$-leveling $\mathcal{Y}$ of $G$ as follows. We set $\mathcal{Y}(v)=0, \mathcal{Y}\left(u_{i}\right)=2 i-1$, and $\mathcal{Y}\left(w_{i}\right)=2 i$.

Extended Degree-3 Spider. Let $G$ be an extended degree-3 spider. Suppose first that $G$ is a 1-connected extended degree-3 spider (see Fig. 5(b)]. We can assume that $G$ is full, i.e., it has the two optional edges (if $G$ is not full we can temporarily add the additional edges to $G$ ). Since $G$ is full it consists of a cycle $C$, plus a vertex $v$ connected to two consecutive vertices of $C$, plus a path $P$ attached to $v$. Denote by $u_{1}, u_{2}, \ldots u_{k}$ the vertices of $C$ (in the order they appear on $C$ ), where $u_{1}$ and $u_{2}$ are the vertices adjacent to $v$. Denote by $w_{1}, w_{2}, \ldots, w_{h}$ the vertices of $P$ (in the order they appear on $P$ ), where $w_{1}$ is the vertex adjacent to $v$. We define a $y$-leveling $\mathcal{Y}$ of $G$ as follows. We set $\mathcal{Y}(v)=0, \mathcal{Y}\left(u_{i}\right)=i$, and $\mathcal{Y}\left(w_{i}\right)=-i$. Suppose now that $G$ is a 2-connected extended degree-3 spider (see Fig. 5(c) . If $G$ is a cycle where one edge has been replaced by a $K_{3}$ gadget, then it is a subgraph of a full 1-connected extended degree-3 spider and therefore a $y$-leveling for $G$ can be defined as in the previous case. If $G$ is a cycle where one edge has been replaced by a $C_{4}$ gadget, then $G$ is the subgraph of a 2-connected extended degree- 3 spider consisting of a cycle where one edge has been replaced by a kite gadget, and a $y$-leveling for $G$ can be defined as in the next case. Thus, suppose that $G$ is a cycle where one edge has been replaced by a kite gadget. In this case $G$ consists of a cycle $C$ plus a vertex $v$ adjacent to three consecutive vertices of $C$. Denote by $u_{1}, u_{2}, \ldots u_{k}$ the vertices of $C$ (in the order they appear on $C$ ), where $u_{1}, u_{2}$, and $u_{3}$ are the vertices adjacent to $v$. We define a $y$-leveling $\mathcal{Y}$ setting $\mathcal{Y}(v)=0$ and $\mathcal{Y}\left(u_{i}\right)=i$.

Generalized Caterpillar. (see Fig. 5(d)). Let $G$ be a generalized caterpillar. $G$ consists of a caterpillar $C$ where some edges of the spine and (possibly) two non spine edges have been replaced by a gadget. We first describe a $y$-leveling $\mathcal{Y}$ for $C$ and then extend it to the vertices of $G$ that are not in $C$. Let $u_{1}, u_{2}, \ldots, u_{k}$ be the vertices of the spine of $C$. If an edge connecting $u_{1}$ to a leaf is replaced by a gadget in $G$, then let this leaf be denoted by $u_{0}$; analogously, if an edge connecting $u_{k}$ to a leaf is replaced by a gadget in $G$, then let this leaf be denoted by $u_{k+1}$. We set $\mathcal{Y}\left(u_{i}\right)=2 i(i=0,1, \ldots, k+1)$. Denote by $w_{i, 1}, w_{i, 2}, \ldots w_{i, h}$ the leaves adjacent to $u_{i}$. We set $\mathcal{Y}\left(w_{i, j}\right)=\mathcal{Y}\left(u_{i}\right)+\frac{j}{h+1}$. Suppose that edge $\left(u_{i}, u_{i+1}\right)(i=0,1, \ldots, k)$ is replaced by a gadget $\gamma$ in $G$. Let $v_{i, 1}, v_{i, 2}, \ldots v_{i, h}(h \geq 1)$ be the vertices of $\gamma$ other than $u_{i}$ and $u_{i+1}$. We set $\mathcal{Y}\left(v_{i, j}\right)=$ $2 i+1+\frac{j-1}{h}($ for $j=1,2, \ldots, h)$. 

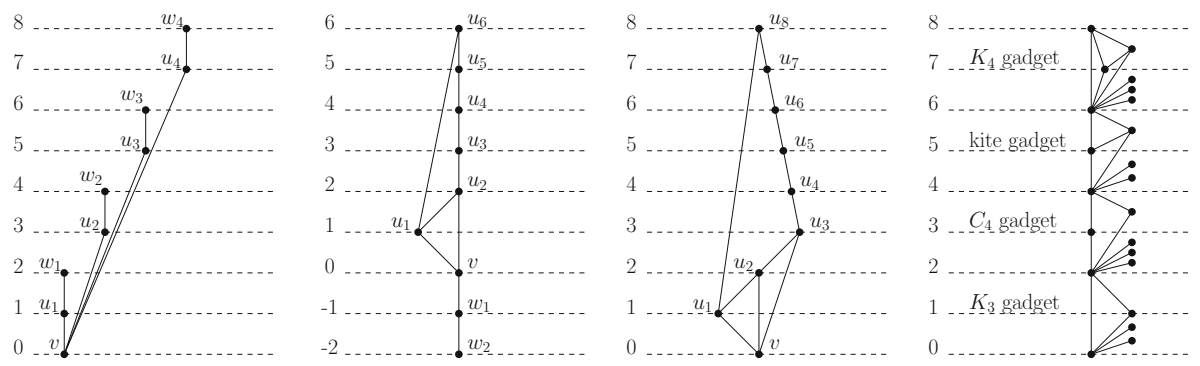

Fig. 5. Illustration of the $y$-levelings described in the proof of Lemma7 (a) A radius-2 star. (b) A full 1-connected extended degree-3 spider. (c) A 2-connected extended degree-3 spider (with an edge replaced by a kite gadget). (d) A generalized caterpillar.

From the discussion above we have $A E P \subseteq E A Q P$. Since the tree $T$ in Fig. 6(a), which is not in $A E P$ [11], is in $E A Q P$ (see Fig. 6(b)], we have $A E P \neq E A Q P$.

The proof of the next lemma is analogous to the one of Lemma 3 .

Lemma 8. Let $G \in E A Q P$. Then $G \in A E Q P$.

It is natural to ask whether $A E Q P$ and $E A Q P$ coincide or not. Also, it is natural to study which families of graphs belong to $A E Q P$ and to $E A Q P$. Theorem 5 answers the first question and gives a result about the second research direction. Theorem 6 summarizes the relationships between $E A P, A E P, E A Q P$, and $A E Q P$.

Theorem 5. All trees are AEQP graphs and there exist trees that are not EAQP graphs.

Sketch of Proof: The fact that every tree is an EAQP graph follows easily from the result in [8]. A tree that is not in EAQP is shown in Fig. 6(c).

Theorem 6. $E A P \subset A E P \subset E A Q P \subset A E Q P$.

In the next theorem we prove that maximal outerpillars are EAQP graphs. An outerplanar graph is called an outerpillar if its weak dual is a caterpillar. An outerpillar is
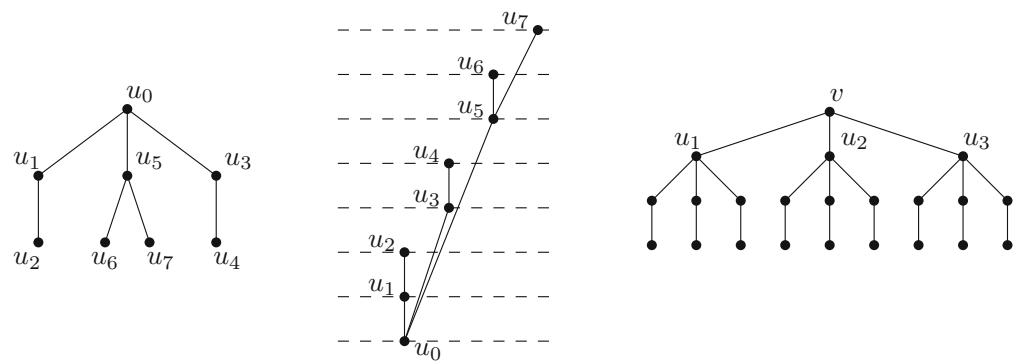

Fig. 6. (a) A tree $T$ that is an EAQP graph but is not an AEP graph. (b) A universal quasi planar $y$-leveling of $T$. (c) A tree that is not an EAQP graph. 
maximal if all its faces have degree three. Let $G=(V, E)$ be a maximal outerpillar and let $G^{*}$ be its weak dual. Let $E_{1} \subseteq E$ be the set of edges of $G$ whose dual edges are in the spine of $G^{*}$. The edges in $E_{1}$ induce a subgraph $C$ of $G$ that is a caterpillar. $C$ is called the backbone caterpillar of $G$. The vertices not in $C$ are called tip vertices of $G$.

\section{Theorem 7. Every maximal outerpillar is an EAQP graph.}

Proof. Let $G$ be a maximal outerpillar. We define a $y$-leveling $\mathcal{Y}$ of $G$ as follows. Let $C$ be the backbone caterpillar of $G$, and let $v_{1}, v_{2}, \ldots, v_{h}$ be the vertices of the spine of $C$ in the order they appear along the spine (for a chosen walking direction). Let $n_{i}$ (with $n_{i} \geq 0$ ) be the number of degree-one vertices of $C$ adjacent to $v_{i}$ (for $i=$ $1,2, \ldots, h)$. We set $\mathcal{Y}\left(v_{1}\right)=0$ and for each vertex $v_{i}(1<i \leq h)$ of $G$ we set $\mathcal{Y}\left(v_{i}\right)=\sum_{j=1}^{i-1} 2\left(n_{j}+1\right)$. Let $w_{i, 1}, w_{i, 2}, \ldots, w_{i, n_{i}}$ be the degree one nodes of $C$ that are adjacent to $v_{i}$ (for $i=1,2, \ldots, h$ ); we set $\mathcal{Y}\left(w_{i, j}\right)=\mathcal{Y}\left(v_{i}\right)+2 \cdot j$. Finally, let $u$ be a tip vertex of $G$; $u$ is adjacent to two vertices $u_{1}$ and $u_{2}$ of $C$. Suppose that $\mathcal{Y}\left(u_{1}\right)<\mathcal{Y}\left(u_{2}\right)$, then we set $\mathcal{Y}(u)=\mathcal{Y}\left(u_{2}\right)-1$

Since the vertices of $G$ have been assigned a different value, $\mathcal{Y}$ is a valid $y$-leveling. Consider now an arbitrary $x$-leveling $\mathcal{X}$. We show that ihs $(\Gamma(\mathcal{X}, \mathcal{Y})) \leq 2$. Let $\ell$ be the stabber at $l$, with $l \in \mathbb{R}$. If $l<0$ or $l>2 n_{c}-2$ (where $n_{C}$ is the number of vertices of $C$ ) then $\ell$ does not intersect any edge. If $l=2 i$ for some $0 \leq i \leq n_{c}-1$, then $\ell$ passes through a vertex $w$ of $C$. If $w$ is a vertex of the spine of $C$, say $v_{j}$ (for $1 \leq j \leq h$ ), then $\ell$ intersects all edges incident to $v_{j}$ and at most two edges both incident to $v_{j-1}$. Thus $\ell$ intersects at most two independent edges. If $w$ is a degree-one vertex of $C$, say $w_{i, j}$, then $\ell$ intersects all edges incident to $w_{i, j}$ and some of the edges incident to $v_{i}$. Also, in this case it intersects at most two independent edges. Consider now the case when $2 i<l<2 i+2$, and let $w_{1}$ and $w_{2}$ be the two vertices of $C$ such that $\mathcal{Y}\left(w_{1}\right)=2 i$ and $\mathcal{Y}\left(w_{2}\right)=2 i+2$. Suppose first that $w_{1}$ is a vertex of the spine of $C$, say $v_{j}(1 \leq j \leq h)$; in this case $w_{2}$ is either the next vertex of the spine of $C$, i.e. $v_{j+1}$, or a degree-one vertex adjacent to $v_{j}$, i.e. $w_{i, 1}$. In both cases $\ell$ intersects some edges incident to $v_{j}$, edge $\left(v_{j-1}, w_{2}\right)$, and, if a tip vertex $u$ adjacent to $v_{j-1}$ and $w_{2}$ exists, at most two edges $\left(v_{j-1}, u\right)$ and $\left(w_{2}, u\right)$; in any case $\ell$ intersects at most two independent edges. Suppose then that $w_{1}$ is a degree-one vertex of $C$, say $w_{j, k}\left(1 \leq j \leq h, 1 \leq k \leq n_{j}\right)$; in this case $w_{2}$ is either another degree-one vertex adjacent to $v_{j}$, i.e. $w_{j, k+1}$, or the next vertex on the spine of $C$, i.e. $v_{j+1}$. In both cases $\ell$ intersects some edges incident to $v_{j}$, the edge $\left(w_{1}, w_{2}\right)$, and, if a tip vertex $u$ adjacent to $w_{1}$ and $w_{2}$ exists, at most the two edges $\left(w_{1}, u\right)$ and $\left(w_{2}, u\right)$; again, $\ell$ intersects at most two independent edges. Hence ihs $(\Gamma(\mathcal{X}, \mathcal{Y})) \leq 2$, which implies that ihs $(G) \leq 2$ and that $G$ is an EAQP graph.

Theorems 4, 5, and 7imply the following.

Corollary 3. Any tree and any cycle have a SGQPE. Any tree and any maximal outerpillar have a $S G Q P E$. 


\section{Discussion and Open Problems}

Several open problems arise from the study of the SGQPE:

Problem 1. Fowler and Kobourov [11] characterized AEP graphs, while in this paper we provided a characterization of EAP graphs. Thus the first obvious open problem is to characterize AEQP and EAQP graphs.

Problem 2. Does every pair of trees (or even every pair of planar graphs) admit a SGQPE? So far we were only able to prove the following.

Theorem 8. There exists a pair of quasi planar graphs that does not admit a SGQPE.

Problem 3. Extend the study of simultaneous embeddability to other families of drawings with forbidden crossing configurations, such as $k$-planar, RAC, LAC, fan-planar, fan-crossing-free-planar drawings.

\section{References}

1. Ackerman, E., Tardos, G.: On the maximum number of edges in quasi-planar graphs. J. of Combinatorial Theory, Series A 114(3), 563-571 (2007)

2. Agarwal, P.K., Aronov, B., Pach, J., Pollack, R., Sharir, M.: Quasi-planar graphs have a linear number of edges. Combinatorica 17(1), 1-9 (1997)

3. Angelini, P., Geyer, M., Kaufmann, M., Neuwirth, D.: On a tree and a path with no geometric simultaneous embedding. J. of Graph Algorithms and Applications 16(1), 37-83 (2012)

4. Bläsius, T., Kobourov, S.G., Rutter, I.: Simultaneous embedding of planar graphs. In: Tamassia, R. (ed.) Handbook of Graph Drawing and Visualization. CRC Press (2014)

5. Brass, P., Cenek, E., Duncan, C.A., Efrat, A., Erten, C., Ismailescu, D., Kobourov, S.G., Lubiw, A., Mitchell, J.S.B.: On simultaneous planar graph embeddings. In: Dehne, F., Sack, J.-R., Smid, M. (eds.) WADS 2003. LNCS, vol. 2748, pp. 243-255. Springer, Heidelberg (2003)

6. Braß, P., Cenek, E., Duncan, C.A., Efrat, A., Erten, C., Ismailescu, D., Kobourov, S.G., Lubiw, A., Mitchell, J.S.B.: On simultaneous planar graph embeddings. Comput. Geom. 36(2), 117-130 (2007)

7. Cabello, S., van Kreveld, M.J., Liotta, G., Meijer, H., Speckmann, B., Verbeek, K.: Geometric simultaneous embeddings of a graph and a matching. J. Graph Algorithms and Applications 15(1), 79-96 (2011)

8. Didimo, W., Kaufmann, M., Liotta, G., Okamoto, Y., Spillner, A.: Vertex angle and crossing angle resolution of leveled tree drawings. Inform. Process. Lett. 112(16), 630-635 (2012)

9. Estrella-Balderrama, A., Fowler, J.J., Kobourov, S.G.: Characterization of unlabeled level planar trees. Computational Geometry 42(6-7), 704-721 (2009)

10. Evans, W., Kusters, V., Saumell, M., Speckmann, B.: Column planarity and partial simultaneous geometric embedding. In: Duncan, C., Symvonis, A. (eds.) GD 2014. LNCS, vol. 8871, pp. 259-271. Springer, Heidelberg (2014)

11. Fowler, J.J., Kobourov, S.G.: Characterization of unlabeled level planar graphs. In: Hong, S.-H., Nishizeki, T., Quan, W. (eds.) GD 2007. LNCS, vol. 4875, pp. 37-49. Springer, Heidelberg (2008)

12. Fox, J., Pach, J., Suk, A.: The number of edges in $k$-quasi-planar graphs. SIAM J. on Discrete Mathematics 27(1), 550-561 (2013)

13. Valtr, P.: On geometric graphs with no $k$ pairwise parallel edges. Discrete \& Computational Geometry 19(3), 461-469 (1998) 